\title{
Studies of Polymer Solvation by Dielectric Relaxation Spectroscopy III: Polyvinyl Pyrrolidone in N-Methyl-2-Pyrrolidone, Ethanediol, Methanol, and Water
}

\author{
E. Dachwitz \\ Institut für Physikalische Chemie der Universität Münster
}

Z. Naturforsch. 45a, 126-134 (1990); received November 2, 1989

The permittivity of the title solutions has been measured at frequencies between some $\mathrm{MHz}$ and $72 \mathrm{GHz}$ at $20^{\circ} \mathrm{C}$. Polyvinyl pyrrolidone of relative molar mass ranging from 1200 to 360000 has been used. Referring to the concentration of monomeric repeat units ( $x=0.65$ maximum) there is no significant chain length effect. The permittivity data are described in terms of discrete Debye type spectral components. Special attention is paid to the contributions ascribable to the solvents, which are discussed with respect to their solvation behaviour. This can be described by a three state solvent model as follows. Besides the "bulk" solvent a further contribution is found in any case which is due to loosely affected (slowed down) solvent. Moreover a part of the solvent appears to be tightly bound to the polymer at high concentrations. All the solvents considered show similar solvation properties, notwithstanding their different tendencies towards selfassociation.

\section{Introduction}

In previous papers [1-3] we have reported on the dielectric relaxation behaviour of some polymer solutions in nonassociating liquids, directing special attention to the solvent relaxation in order to gain information on solvation. A simple two state model of solvent relaxation was found to be adequate for the systems regarded, which means that unaffected "bulk" solvent and affected "solvating" solvent are distinguishable by their relaxation times. These studies left some problems in need of clarification. First, the applicability of the simple solvation model is subject to question for higher concentrations of the polymer, the investigation of which was, however, denied for experimental reasons (high viscosities). Second, self-associating solvents (e.g. water) were not yet regarded. Here the question arises as to whether these might exhibit a similarly simple solvation behaviour.

In the present communication, we report on measurements concerning these topics. The polymer considered is polyvinyl pyrrolidone (PVP), which was already used previously $[1,2]$. Concerning the first point, it is studied with $\mathrm{N}$-methyl-2-pyrrolidone (MPy) as nonassociating solvent as in [1] but now up to the highest concentration which we could handle with the apparatus modified for that purpose. Concerning the second point, PVP was studied with

Reprint requests to Dr. E. Dachwitz, Institut für Physikalische Chemie der Universität, Schloßplatz 4/7, D-4400 Münster (FRG). ethanediol $\left(\mathrm{Et}(\mathrm{OH})_{2}\right)$, methanol $(\mathrm{MeOH})$ and water $\left(\mathrm{H}_{2} \mathrm{O}\right)$ (already regarded in $\left.[4,5]\right)$ as solvents in order to get information on solvation by these liquids which are capable of self-association. Results and discussion will be given for the nonassociating and self-associating solvents in two separate sections.

By the way, we shall regard the influence of the polymer chain length on solvation behaviour by using PVPs of different relative molar masses.

\section{Experimental}

The solvents were obtained from Aldrich and were used without further purification. High purity PVP with a relative molar mass of 1200 was kindly provided by the Kunststofflabor of BASF/Ludwigshafen. PVP with relative molar masses of 10000,24000 , 40000 and 360000 were from Aldrich. Solutions in MPy were prepared with all these different PVPs. The highest concentration was a mole fraction $x_{\mathrm{mPVP}}=$ 0.65 as related to monomeric units. The solutions in $\mathrm{Et}(\mathrm{OH})_{2}$ and $\mathrm{MeOH}$ were prepared with PVP 10000 and 40000 up to a mole fraction of $x_{\mathrm{mPVP}}=0.4$, the solutions in $\mathrm{H}_{2} \mathrm{O}$ with PVP 1200 and 40000 up to a mole fraction of $x_{\mathrm{mPVP}}=0.3$.

The complex permittivity $\varepsilon^{*}(\omega)=\varepsilon^{\prime}(\omega)-i \varepsilon^{\prime \prime}(\omega)$ was measured at $20^{\circ} \mathrm{C}$ in the frequency range between $2 \mathrm{MHz}$ and $72 \mathrm{GHz}$ at up to 14 fixed frequencies. Since the dielectric relaxation spectrum of liquids contains no finer structures than that of a Debye-type spectral function, those measurements at several fixed

0932-0784 / $90 / 0200-0126 \$ 01.30 / 0$ - Please order a reprint rather than making your own copy. 
points distributed over the whole frequency range are sufficient to satisfactorily describe the relaxation behaviour.

The experimental uncertainty of $\varepsilon^{\prime \prime}$ is less than $5 \%$, $\varepsilon^{\prime}(\omega)$ (which will be considered only occasionally) is somewhat more uncertain. In addition, the density and the viscosity (after Ostwald) for some solutions were determined.

\section{Results and Discussion}

\subsection{Data Treatment and Description of Results}

Both parts of the complex permittivity $\varepsilon^{*}(\omega)=$ $\varepsilon^{\prime}(\omega)-i \varepsilon^{\prime \prime}(\omega)$ contain in principle the same information (Kramers-Kronig theorem). Because of the immediate physical meaning of $\varepsilon^{\prime \prime}(\omega)$, which is related to the polarization fluctuation spectrum of the dielectric sample, we shall preferably consider that quantity. Since we are interested in dielectric relaxation processes only, its measured value has to be corrected for the conductivity contribution $\varepsilon_{\mathrm{c}}^{\prime \prime}(\omega)=\varkappa / \varepsilon_{0} \omega\left(\varepsilon_{0}\right.$ : electric field constant; $x$ : conductivity). The remaining dielectric loss $\varepsilon^{\prime \prime}(\omega)$ may then be represented as absorption curve, which in the simplest case is of Debye type. Generally, the $\varepsilon^{\prime \prime}(\omega)$ data will be fitted by presupposed functions corresponding to discrete or continuous relaxation time distributions. The real part $\varepsilon^{\prime}(\omega)$ is used to check the analysis.

In order to introduce the functions and the fitting procedure used here, it may be helpful to consider an illustrative example. Figure 1 shows the absorption of a concentrated MPy/PVP solution in comparison with that of the pure solvent MPy. While the MPy data can satisfactorily be fitted by one Debye curve, the question arises as how to describe the extremely broad absorption of the solution. Several functions based on continuous relaxation time distributions (Cole-Davidson, Havriliak-Negami, Jonscher, etc.) as often applied in polymer research, fail in describing the experimental data by one function, i.e. without allowing for the superposition of several of those functions. Since in that situation it would not only be difficult to obtain reliable parameters but also to interpret them physically, we shall preferably use a discrete relaxation time distribution, viz. a sum of Debye type spectral components $C_{i}$ according to

$$
\varepsilon^{\prime \prime}(\omega)=\sum_{i} S_{i} \frac{\omega \tau_{i}}{1+\omega^{2} \tau_{i}^{2}} .
$$

It is feasible that some of those spectral components may reasonably be considered as coherent so that they are describable also by a continuous relaxation time distribution. Therefore the description after (1) is formal and does not necessarily mean that each component should have its separate physical meaning.

The example in Fig. 1 needs a minimum number of six terms according to (1) for a satisfactory fit within the $5 \%$ error range. Their parameters $\tau_{i}$ (relaxation time) and $S_{i}$ (relaxation strength or dispersion step) can be varied to a certain extent which, however, is fairly restricted due to the shape of the absorption curve.

The general data treatment for less concentrated solutions is now based on these results. We allow for the same number of spectral components as in the high concentration case and follow up the development of their parameters on decreasing the polymer content. Arriving at the semidilute regime, we obtain in that manner still minor terms which were not revealed in the previous work [1] dealing only with semidilute solutions.

\subsection{Methylpyrrolidone-Polyvinyl Pyrrolidone Solutions}

Results

The relaxation parameters for the MPy/PVP systems obtained in the manner described in the preceding paragraph are given graphically in Figure 2.

\section{Molar mass effects}

In Fig. 2 solutions of PVP 1200, 10000,24000 , 40000 and 360000 are included without indicating the different molar masses by distinguishing symbols. The reason is that on the scale of the monomeric PVP mole fraction, $x_{\mathrm{mPVP}}$, no significant difference of the relaxation parameters of different PVPs can be ascertained. This holds also for the solvents regarded subsequently. Throughout this paper, it is therefore not necessary to refer to the molar mass of the polymer. A direct conclusion from that finding is that in attempts to assign spectral components to fluctuational processes, very large scale motions (i.e. those of whole polymer molecules) need not be taken into consideration. 


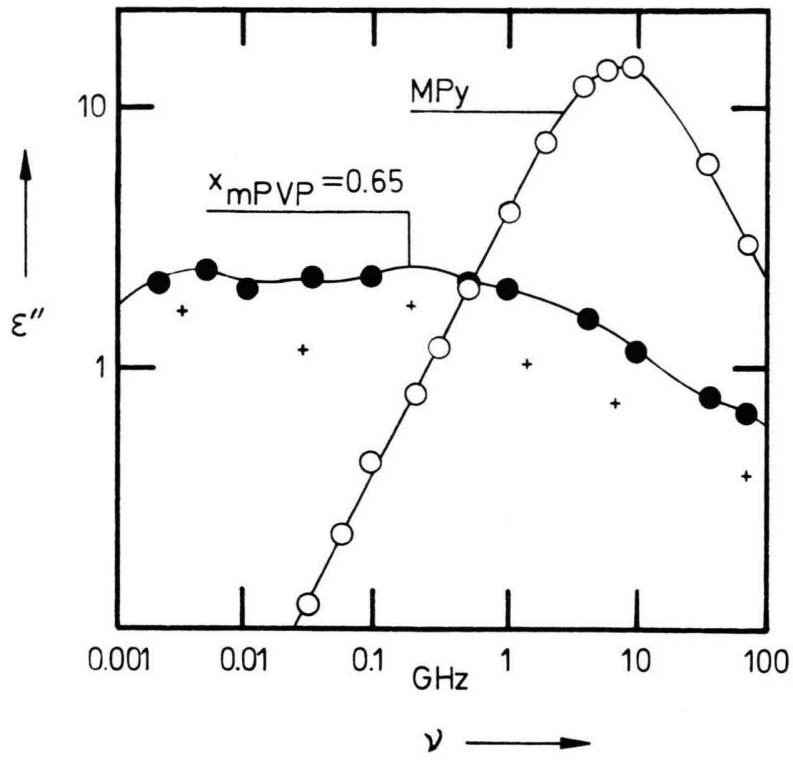

Fig. 1. Log-log plot of the imaginary part $\varepsilon^{\prime \prime}$ of the permittivity against frequency $v$ for pure MPy and for a concentrated solution of PVP 1200 with that solvent.

\section{Assignment of Spectral Components}

As a noticeable feature, Fig. 2 shows that all the relaxation times are practically independent of the polymer concentration (perhaps except for $\tau_{1}$ ), though at higher concentrations the viscosity is not only enhanced by up to two orders of magnitude but also increases (on the $x_{\mathrm{mPVP}}$ scale) the steeper the higher the PVP molar mass. This indicates that all spectral components are due to local motions within a microheterogeneous solution.

The component $C_{2}$ is obviously due to the solvent ( $\tau_{2}$ is about the pure MPy relaxation time, and $S_{2}$ starts from the pure MPy value). The remaining components initially increase as the polymer content, thus must be related to the polymer in some way. The component $C_{1}$ (which together with $C_{2}$ is predominating) was already observed in previous work $[1,3]$ and was assigned to "solvating" MPy molecules. The question remains as to wether the additional components now revealed can be assigned as unequivocally as the main components to either the solvent or the solute. For that purpose it is helpful to consider particularly the relaxation strengths.

According to simple dielectric models the relaxation strength originating in the motion of certain polar moieties is proportional to their concentration. We assume this to be applicable at least in an approx-

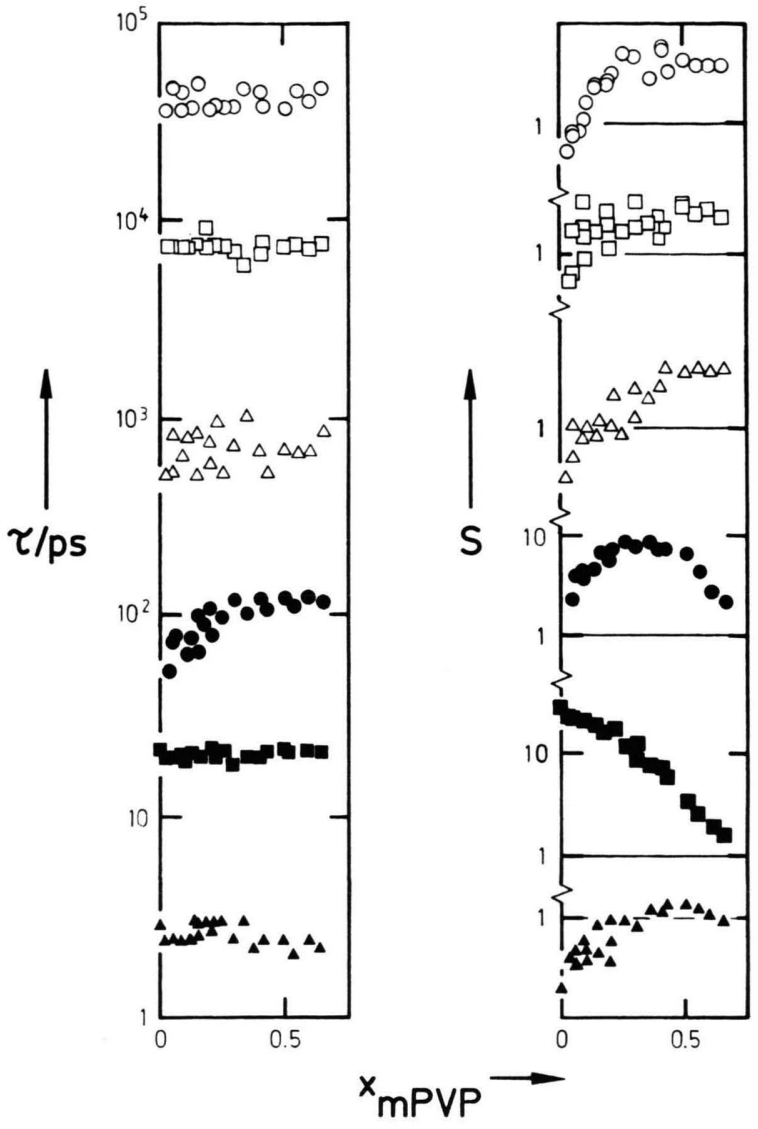

Fig. 2. Relaxation parameters $\tau_{i}$ and $S_{i}$ (log scales) against PVP concentration (mole fraction $x_{\text {pyp }}$ of monomeric units) for PVP-MPy solutions. The $S_{i}$ scales are shifted and ordered according to the respective relaxation times $\tau_{i}$. Notation of spectral components: $C_{1} \bullet ; C_{2} \mathbf{\bullet} ; C_{3} \mathbf{\Delta} ; C_{4} \Delta ; C_{5} \square ; C_{6} \circ$.

imate manner, so that the "dilution" of the solvent MPy by adding the polymer in the absence of other effects should result in an "expected" relaxation strength

$$
S_{\mathrm{MPy}}^{\mathrm{e}}=\frac{c_{\mathrm{MPy}}}{c_{\mathrm{MPy}}^{*}} S_{\mathrm{MPy}}^{*}
$$

(the asterisk indicating the pure state). Experimentally it is found that $S_{2}<S_{\mathrm{MPy}}^{\mathrm{e}}$ while at least in the lower concentration region $S_{1}+S_{2} \approx S_{\mathrm{MPy}}^{\mathrm{e}}$, so that $C_{1}$ and $C_{2}$ together are likely to comprise the whole solvent relaxation. The constancy is somewhat better if one adds $S_{3}$, too. The ratio $\sigma_{\mathrm{I}}=\sum_{i=1}^{3} S_{i} / S_{\mathrm{MPy}}^{\mathrm{e}}$ is plotted in Figure 3. Its decrease at higher polymer contents indicates a "disappearance" of solvent molecules. This cannot be compensated by including further spectral 


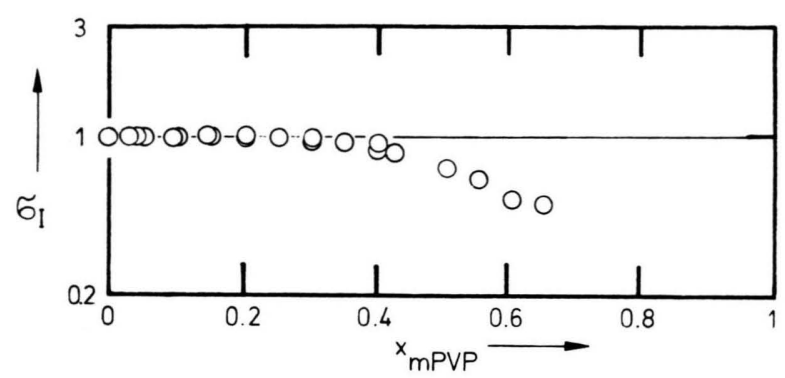

Fig. 3. Total relative relaxation strength $\sigma_{\mathrm{I}}=\sum_{i=1}^{3} S_{i} / S_{\mathrm{MPy}}^{\mathrm{e}}(\log$ scale) ascribed to the solvent against polymer mole fraction $x_{\mathrm{mPVP}}$ for PVP-MPy systems.

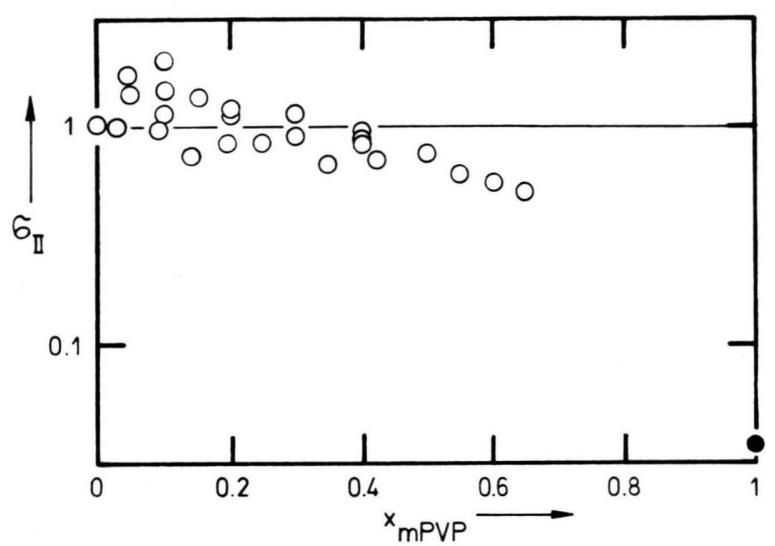

Fig. 4. Total relative relaxation strength $\sigma_{\mathrm{II}}=\sum_{i=4}^{6} S_{i} / S_{\mathrm{mPVP}}^{\mathrm{e}}$ (log scale) ascribed to the polymer against polymer mole fraction $x_{\text {mPVP }}$ for PVP-MPy systems. Open symbols: this work; full point: solid PVP, estimated after [6].

components, since this would yield unreasonable high ratios in the semidilute region.

To a certain degree, the approximate nature of (2) may account for the decrease of $\sigma_{\mathrm{I}}$. In fact, an estimate of the relaxation strength and its variation on dilution according to the Onsager and related theories leads to a smaller $S_{\mathrm{MPy}}^{\mathrm{e}}$ than given by (2). This reduction, however, amounts to only some percent in $\sigma_{\mathrm{I}}$, which still leaves a significant difference to the $\sigma_{\mathrm{I}}$ values observed at higher concentrations. It should be mentioned here that the liquid-liquid mixture system $\mathrm{MPy} /$ polyethylene glycol shows $\sigma_{\mathrm{I}} \approx 1$ over the whole mixture range [3], so that also with respect to this system the present finding of $\sigma_{\mathrm{I}}<1$ seems to be a noteworthy peculiarity.

One may now conclude from the relaxation strengths that $C_{1}$ and $C_{2}$ (and possibly $C_{3}$, too) should be ascribed to motions of solvent molecules, while $C_{4}$ to $C_{6}$ should consequently be due to motions within the polymer. This assignment is corroborated by the magnitude of the relaxation times $\tau_{4}$ to $\tau_{6}(800$ to $32000 \mathrm{ps}$, respectively), which are by far longer than known for such small molecules as MPy in low molecular mass liquid environments.

It should be mentioned that there remain doubts on the assignment of the high frequency component $C_{3}$, which is, however, a formally inevitable component. It follows from the results on aqueous systems reported below that $C_{3}$ cannot be generated by residual water, as might be suspected. Because of the short relaxation time the component $C_{3}$ is likely to originate in the motion of small moieties. Possibly it reflects special solute-solvent interactions so that an assignment to either of them would be meaningless.

\section{Polymer Relaxation Contribution}

The side groups of PVP have about the same dipole moment as the MPy molecules. Assuming that the side groups move as independently tumbling entities, the corresponding relaxation strength for that hypothetical case can easily be estimated since the total dipole density (MPy plus PVP) is practically independent of the solution composition (though the mass density slightly alters). The expected PVP relaxation strength $S_{\mathrm{mPVP}}^{\mathrm{e}}$ is (by putting the hypothetical $S_{\mathrm{mPVP}}^{*}$ $=S_{\mathrm{MPy}}^{*}$ ) approximately

$$
S_{\mathrm{mPVP}}^{\mathrm{e}}=\frac{c_{\mathrm{mPVP}}}{c_{\mathrm{mPVP}}^{*}} S_{\mathrm{MPy}}^{*}
$$

(“"m" referring to monomeric repeat units).

The total normalized polymer relaxation strength $\sigma_{\mathrm{II}}=\sum_{i=4}^{6} S_{i} / S_{\mathrm{mPVP}}^{\mathrm{e}}$ is given graphically in Figure 4 . It behaves similarly to the corresponding solvent ratio, Figure 3. In spite of the scatter the figure suggests that $\sigma_{\text {II }}>1$ at low concentrations while again dipoles seem to "disappear", viz. $\sigma_{\mathrm{II}}<1$, with increasing concentration. Consequently the assumption of independently moving side groups cannot be correct for the whole concentration range. On increasing the polymer content they obviously become orientationally correlated such that a mean compensation of moments results. Actually, the total relaxation strength of solid PVP is estimated from [6] to be about 50 times less than $S_{\mathrm{mPVP}}^{\mathrm{e}}$ after (3) (full point in Figure 4). 


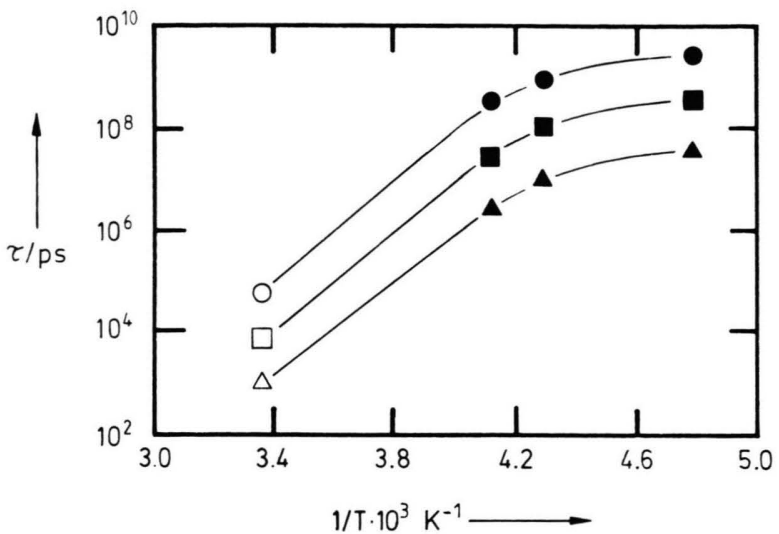

Fig. 5. Comparison of relaxation times of solid PVP at low temperatures (full points, obtained by reanalyzing the results from [6]) and of $\tau_{4}$ to $\tau_{6}$ of the present PVP-MPy solutions (open symbols).

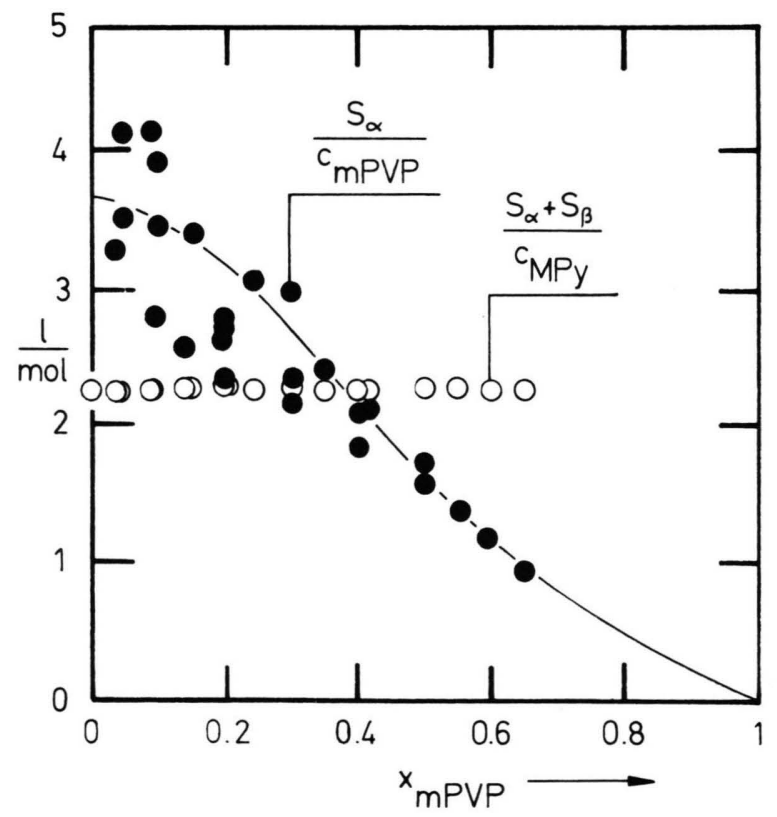

Fig. 6. Solvent relaxation contributions in PVP-MPy systems. Normalized relaxation strength $S_{\alpha}$ (affected solvent) and $S_{\alpha}+S_{\beta}$ (total solvent contribution) are given by full and open symbols, respectively, against polymer mole fraction $x_{\mathrm{mPVP}}$. See text for the definition of $S_{\alpha}$ and $S_{\beta}$.

To get a further comparison with solid PVP, we have reanalyzed low temperature literature data [6] in terms of (1) and have obtained three spectral components which correspond to our present components $C_{4}$ to $C_{6}$, as can be seen from the temperature dependence of their relaxation times depicted in Figure 5. This indicates once more that the present components $C_{4}$ to $C_{6}$ comprise essentially the polymer relaxation.
In order to further support that conclusion some PVP solutions in the practically nonpolar solvents 1,4dioxane and 1,4-difluorobenzene were investigated. These solutions exhibit just the spectral components termed here $C_{4}$ to $C_{6}$. The relaxation times are similar to $\tau_{4}$ to $\tau_{6}$ of the MPy-PVP system, only the dispersion strengths differ.

The fluctuations generating $C_{4}$ to $C_{6}$ are very likely to be segmental motions. No further discussion of them will be given since the main interest of this work concerns the solvent rather than the solute relaxational behaviour.

\section{Solvent Relaxation Contribution}

The simple solvation model used in [1-3] for semidilute solutions takes into account two kinds of solvent molecules. Those in the vicinity of the polymer molecules are "affected" and are "bound" in gel particles, their tumbling motion is slowed down. Molecules without direct contact to the polymer, on the other hand, behave as "free" ones. The applicability of this picture points to a microheterogenity of the solution. The ratio of affected solvent molecules to polymer repeat units (the solvation number) depends on the concentration, since otherwise in concentrated solution all solvent molecules would be bound so that no spectral component due to "free" molecules should be left, in contrast to the experimental results. Thus an equilibrium between "affected" $(\alpha)$ and "free" $(\beta)$ solvent molecules should be assumed. Let this be formulated as [3]

$$
\mathrm{A}+\mathrm{B} \rightleftarrows \mathrm{AB},
$$

where A is a section of the polymer chain (not necessarily a repeat unit) which affects just one solvent molecule B. By simple assumptions [3] the relaxation strengths can be related to the model concentrations $c_{\mathrm{B}}$ and $c_{\mathrm{AB}}: S_{\beta} \sim c_{\mathrm{B}}$ (free solvent) and $S_{\alpha} \sim c_{\mathrm{AB}}$ (affected solvent). Assuming that the observed relaxation strengths relate to affected and free solvent as $S_{\alpha}=S_{1}$ and $S_{\beta}=S_{2}$ it is found hardly possible to describe the observed dependence of $S_{1}$ on $x_{\mathrm{mPVP}}$ by the equilibrium (4). If, however, the "disappeared" solvent (see Fig. 3) is added to the affected solvent portion, viz. $S_{\alpha}=\left(S_{\mathrm{MPy}}^{\mathrm{e}}-S_{2}\right)$, (4) can be used to describe that dependence fairly well. This is shown in Fig. 6, where the normalized relaxation strength $S_{\alpha} / c_{\mathrm{mPVP}}$ is given together with a fitting curve according to (4). Figure 6 also shows the normalized relaxation strength 
$\left(S_{\alpha}+S_{\beta}\right) / c_{\mathrm{MPy}}$ (with $S_{\beta}=S_{2}$ ). The ratio of both these quantities gives the solvation number

$$
Z=\frac{S_{\alpha}}{c_{\mathrm{mPVP}}}\left(\frac{S_{\alpha}+S_{\beta}}{c_{\mathrm{MPy}}}\right)^{-1} .
$$

Here in the dilute solution limit there is $Z_{0} \approx 2$. On increasing the polymer content, $Z$ decreases gradually.

In concluding it seems possible to use the solvation model originally deduced from semidilute solutions [1] also for highly concentrated MPy-PVP solutions if one takes into account a certain fraction of dielectrically "disappeared", viz. strongly influenced solvent. With this modification, it becomes in fact a three state rather than a two state model. It has to be considered in a dynamical sense, meaning that one certain molecule can be pictured to experience different states in the course of time, tumbling with different correlation times in each state. Assuming slow exchange, such a motion will cause a correlation function consisting of superimposed exponentials, corresponding to a dielectric relaxation behaviour as found in this experiments. A similar two state model including exchange was presented in [7] in relation to the problem of H-bonding.

\subsection{Ethanediol or Methanol \\ or Water-Polyvinyl Pyrrolidone Solutions}

\section{Results}

The results given in this section are obtained by some reference to those of the preceding section as follows. Considering the $\mathrm{H}_{2} \mathrm{O}-\mathrm{PVP}$ system as an example, a formal fit for concentrated solutions is possible with 5 terms [8]. The MPy-PVP results presented above (Fig. 2), on the other hand, were characterized by 6 terms, their $\tau_{i}$ values being practically independent of concentration. Concerning the polymer this means little influence of the environment on the relaxation times $\tau_{4}$ to $\tau_{6}$, so that it might be permissible to assume about the same polymer relaxation times even on changing the solvent. Accordingly one may adopt $\tau_{4}$ to $\tau_{6}$ from the MPy-PVP system as approximate starting values for the fitting procedure of the $\mathrm{H}_{2} \mathrm{O}$ PVP data. This model assumption results in a six term fit which differs from the formal five term fit mentioned above essentially in that a component around $3700 \mathrm{ps}$ is now split into two components $C_{4}$ and $C_{5}$. Note that the total relaxation strength to be ascribed

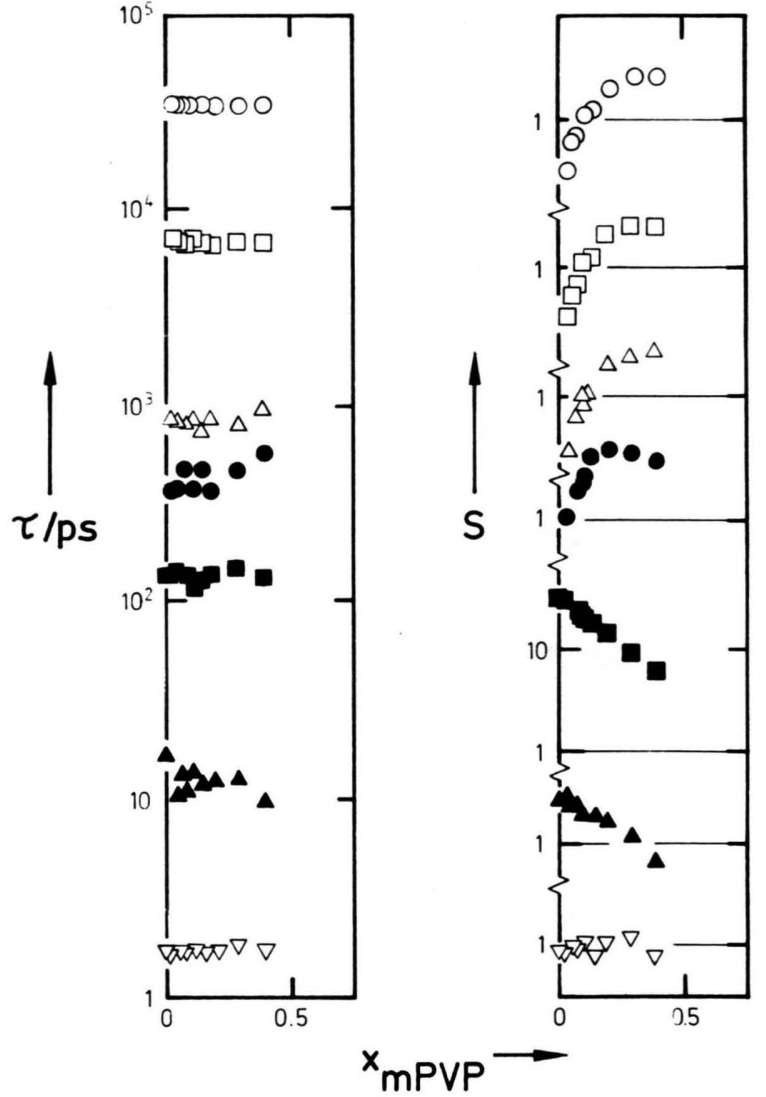

Fig. 7. Relaxation parameters $\tau_{i}$ and $S_{i}$ (log scales) against PVP mole fraction $x_{\text {mPyP }}$ for PVP-Et $(\mathrm{OH})_{2}$. Scales and symbols as in Fig. 2; additionally: $C_{7} \nabla$.

to the polymer appears practically unchanged regardless of the number of terms. Now it is not only the consistency with the MPy-PVP results but also the somewhat closer fit which leads us to apply the modified procedure to all $\mathrm{Et}(\mathrm{OH})_{2}-\mathrm{PVP}, \mathrm{MeOH}-\mathrm{PVP}$ and $\mathrm{H}_{2} \mathrm{O}-\mathrm{PVP}$ systems considered in this section. Results obtained in that manner are represented in Figures 7-9. It should be mentioned that there is again no influence of chain length, so that points belonging to PVPs of different molar mass are not distinguished in Figures 7-9.

According to the above assumptions, the low frequency spectral components $C_{4}$ to $C_{6}$ are again assigned to the polymer, while the other components should be due to the solvent. These are three components for $\mathrm{H}_{2} \mathrm{O}$ and $\mathrm{MeOH}$ but four in the case of $\mathrm{Et}(\mathrm{OH})_{2}$. In the latter case the spectral components $C_{1}$ and $C_{4}$ may be combined within experimental uncertainty limits in one spectral component the inter- 


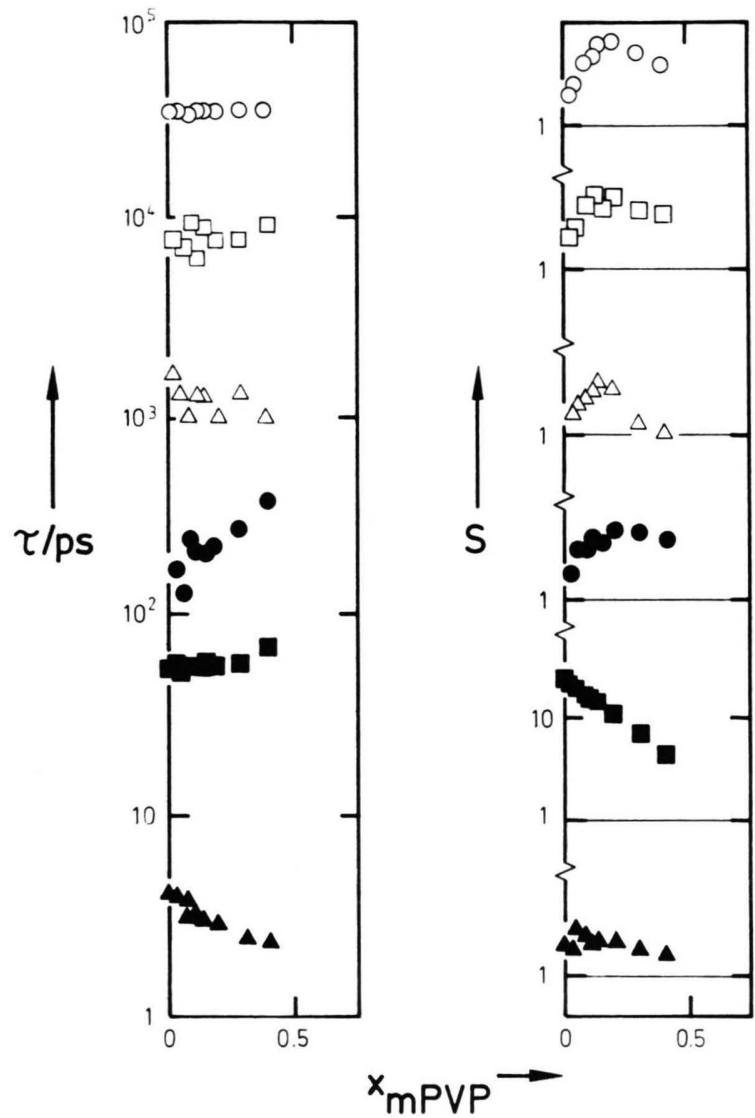

Fig. 8. Relaxation parameters $\tau_{i}$ and $S_{i}$ (log scales) against PVP mole fraction $x_{\text {mPVP }}$ for PVP-MeOH. Scales and symbols as in Figure 2.

pretation of which, however, is more difficult since the relation to pure $\operatorname{Et}(\mathrm{OH})_{2}$ parameters gets lost.

\section{Polymer Relaxation Contribution}

A striking feature of Figs. 8 and 9 is the steep increase of the polymer relaxation strengths $S_{4}$ to $S_{6}$ on increasing the polymer concentration, which is clearly greater than in the case of MPy-PVP (Fig. 2) and also of $\mathrm{Et}(\mathrm{OH})_{2}$ - PVP (Figure 7). The total polymer relaxation strengths $\sum_{i=4}^{6} S_{i}$ are given in Fig. 10 to illustrate this. (That figure includes additional data for the system 1,4-difluorobenzene-PVP as an example with a total polymer relaxation strength clearly less than for MPy-PVP.) Two possible explanations of the solvent effect are (i) conformational changes of the polymer such that its effective dipole moment per repeat unit is altered; (ii) a "strong" solute-solvent interaction which

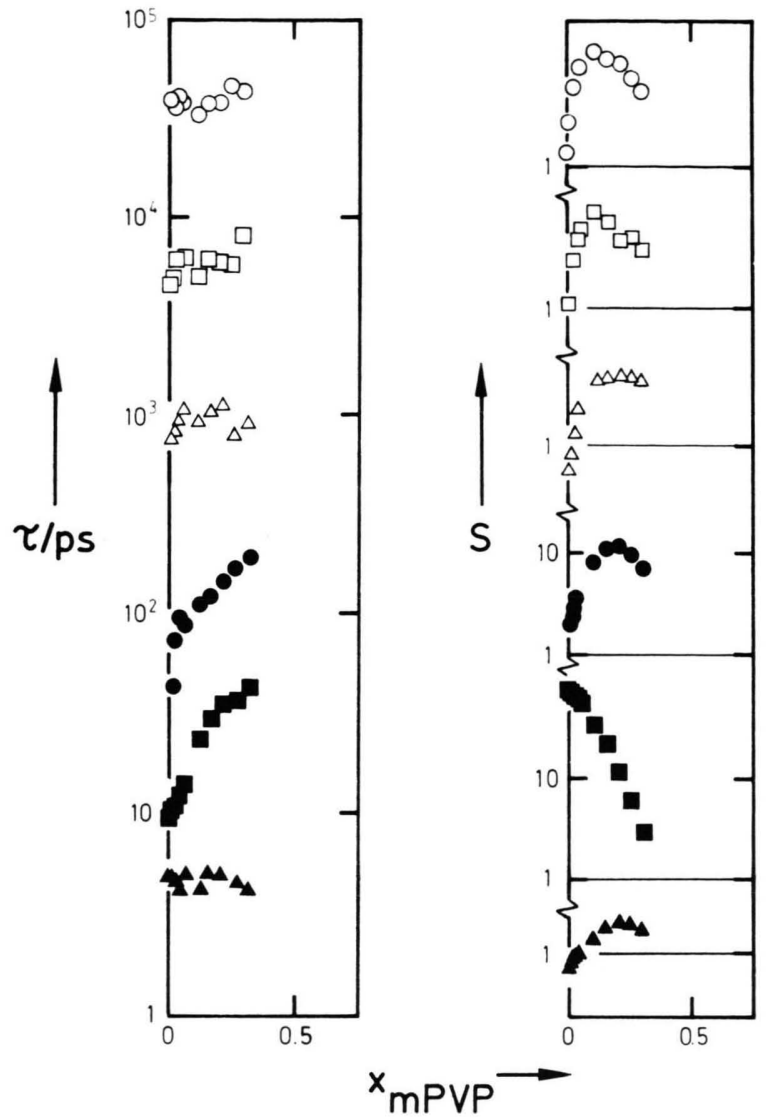

Fig. 9. Relaxation parameters $\tau_{i}$ and $S_{i}$ (log scales) against PVP mole fraction $x_{\text {Pyp }}$ for PVP- $\mathrm{H}_{2} \mathrm{O}$. Scales and symbols as in Figure 2.

causes association of solvent molecules (in particular of $\mathrm{MeOH}$ and $\mathrm{H}_{2} \mathrm{O}$ ) to the polymer side groups and enhances the resulting moment.

If (i) should be the case, a preferential parallel order of side group moments must occur in aqueous or alcoholic solution since for dilute MPy-PVP solutions it was inferred above that the side groups are approximately uncorrelated in their orientation. Similarly (ii) would imply a constructive addition of PVP side group and solvent moments for the $\mathrm{H}_{2} \mathrm{O}$ and $\mathrm{MeOH}$ systems. Since the enhancement effect with $\mathrm{Et}(\mathrm{OH})_{2}$ appears to be negative, one may speculate if this points to an association of $\mathrm{Et}(\mathrm{OH})_{2}$ such that two PVP side groups are involved with partial moment compensation. In the 1,4-difluorobenzene-PVP solutions a compensation of partial moments is obvious, too.

A decision in favour of one of these possibilities cannot be drawn from merely the present results. The 


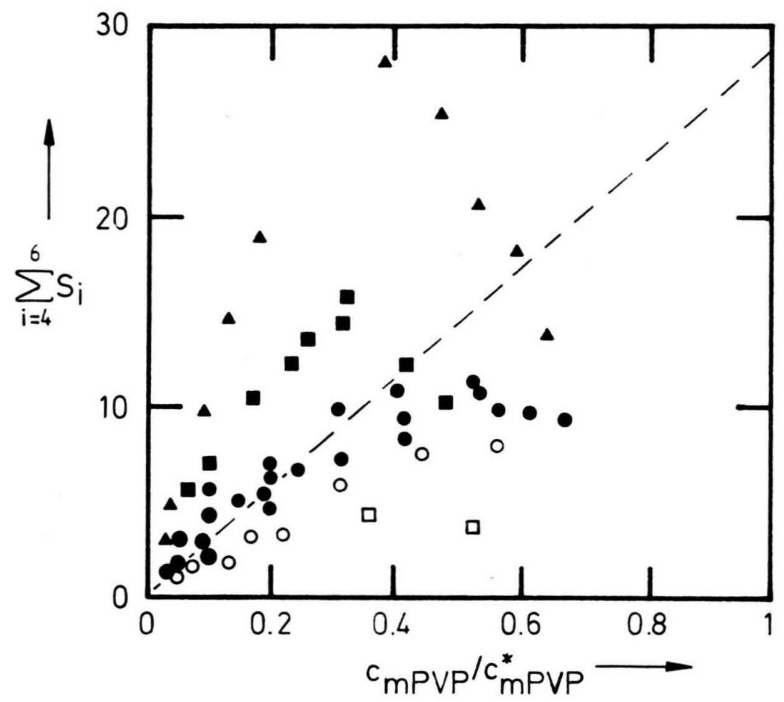

Fig. 10. Total polymer relaxation strength $\sum_{i=4}^{6} S_{i}$ against relative polymer concentration (the asterisk indicates pure PVP) for PVP in different solvents: MPy $\bullet ; \mathrm{Et}(\mathrm{OH})_{2} \mathrm{O} ; \mathrm{MeOH} \mathbf{~}$; $\mathrm{H}_{2} \mathrm{O} \Delta$; 1,4-difluorobenzene $\square$.

pronounced reduction of the total polymer relaxation strength in the nonpolar solvent 1,4-difluorobenzene points to case (i). It is, on the other hand, known from the dielectric relaxation behaviour of pyrrolidone in mixtures with water and alcohols that definite heteroassociates are formed [9]. Since the side group of PVP resembles the pyrrolidone molecule, hetero-association, viz. case (ii), appears to be also a very likely explanation of the differences found in comparison to the MPy solutions. If this should be the case, the magnitude of relaxation times indicates that the tumbling motion of associated solvent molecules is governed by the motion of polymer segments.

\section{Solvent Relaxation Contribution}

The spectral components found for the pure solvents do also persist in the solutions, resembling the behaviour of these solvents with small pyrrolidone type molecules as solutes [9]. In all cases the predominant relaxation contribution $\left(C_{2}\right.$ in Figs. $\left.7-9\right)$ can be attributed to the self-associated part of the solvent. The minor contribution $\left(C_{3}\right.$ in Figs. 7-9) can be ascribed to low order self-associates or monomeric molecules. The third region, which is only obtained for
$\mathrm{Et}(\mathrm{OH})_{2}$, may be caused by intramolecular motions $\left(C_{7}\right.$ in Figure 7$)$.

As a conspicuous feature, an additional spectral component $C_{1}$ appears with increasing PVP concentration in all cases, which is not found in the pure solvents. Its relaxation strength $S_{1}$ initially increases, passes through a maximum and decreases subsequently. This behaviour is closely similar to that of the MPy-PVP system. Therefore $C_{1}$ should be ascribed to the polymer affected solvent molecules as before. Again these molecules are slowed down in their motion but are not bound immovably to the polymer. The question remains as to which degree these molecules might still participate in self-association. It is not possible to estimate a solvation number as before without assuming a Kirkwood $g$-factor for the solvating portion of the solvent. We can, however, state as a qualitative finding that the solvation behaviour of the solvents considered in this section is similar to that of MPy notwithstanding the fact of self-association within at least the bulk solvent.

Some special remarks should be made on the $\mathrm{H}_{2} \mathrm{O}$ PVP system, which differs from all the other systems in the considerable increase of the relaxation times $\tau_{1}$ and $\tau_{2}$ with increasing PVP concentration. A similar behaviour was found for aqueous solutions of several pyrrolidones [9]. It points to a structure forming effect by an increase of the lifetime of associated water domains. Other formal descriptions of the water relaxation are possible which, however, lead qualitatively to equivalent conclusions. Kaatze [4] has characterized the water relaxation by a Cole-Cole relaxation time distribution. He found the Cole-Cole parameter $h$ to increase with increasing PVP concentration and to pass through a maximum at $x_{\mathrm{mPVP}} \approx 0.15$. At just this concentration the dispersion steps $S_{1}$ and $S_{2}$ of our description have equal magnitude, thus the broadening of the combined relaxation region has a maximum. At higher concentrations there is $S_{1}>S_{2}$ and the broadening is reduced again. The relaxation times reported in [4] increase with increasing PVP concentration in accordance with our experiments. In [4] the results are discussed with a solvation model inferring that "free" water exists only up to $x_{\mathrm{mPVP}} \approx 0.08$, in contrast to our experiments which indicate "free" water still at higher concentrations.

It should finally be mentioned that the measurements above $50 \mathrm{MHz}$ in [4] did not reveal the low frequency polymer contribution but that $\varepsilon^{\prime}(\omega)$ results on $\mathrm{H}_{2} \mathrm{O}$-PVP [10] obtained also at lower frequencies 
exhibit a dispersion region in the 1 to $10 \mathrm{MHz}$ range (at $25^{\circ} \mathrm{C}$ ) ascribable to the polymer, which is in accord with our findings.

\section{Conclusions}

The "strong" interactions of MPy with PVP as suspected from the results in the first section of this work are also evidenced in the second section for the solvents capable of $\mathrm{H}$-bonding. A spectral component $C_{1}$ to be ascribed to "loosely" affected solvent molecules (only slowed down in their tumbling motion) can be ascertained in all cases. Thus it turns out that the solvation behaviour of all the four liquids is similar in

[1] E. Dachwitz, Z. Naturforsch. 43a, 285 (1988).

[2] M. Rohmann, E. Dachwitz, and M. Stockhausen, Coll. Polymer Sci. (in press).

[3] H. Utzel, E. Wessling, E. Dachwitz, and M. Stockhausen, Coll. Polymer Sci. (in press).

[4] U. Kaatze, Adv. Molec. Relax. Interact. Proc. 7, 71 (1975) and Prog. Coll. Polymer Sci. 65, 214 (1978).

[5] R. Pottel, E. Asselborn, R. Eck, and V. Tresp, Ber. Bunsenges. Phys. Chem. 93, 676 (1989).

[6] S. K. Jain and C. P. Johari, J. Phys. Chem. 92, 5851 (1988). a qualitative manner, notwithstanding their different tendency towards self-association.

Also the polymer relaxational behaviour is qualitatively the same in the solvents regarded. A probable conformational change on increasing concentration such that the apparent dipole moment changes seems to be a common feature.

A modified (three state) solvation model is thus appropriate regardless of association peculiarities.

\section{Acknowledgements}

Thanks are due to Prof. M. Stockhausen for many helpful discussions, to the Deutsche Forschungsgemeinschaft for financial support and to BASF/Ludwigshafen for supplying PVP 1200.

[7] M. Stockhausen and E. Dachwitz, Z. Naturforsch. 39a, 646 (1984).

[8] E. Dachwitz, F. Parak, and M. Stockhausen, Ber. Bunsenges. Phys. Chem. (in press).

[9] E. Dachwitz and M. Stockhausen, Ber. Bunsenges. Phys. Chem. 91, 1347 (1987).

[10] E. H. Grant, V. E. R. McClean, N. R. V. Nightingale, R. J. Sheppard, and M. J. Chapman, Bioelectromagnetics 7, 151 (1986). 